\title{
ON THE SEGAL CONJECTURE FOR $Z_{2} \times Z_{2}$
}

\author{
DONALD M. DAVIS ${ }^{1}$
}

\begin{abstract}
The Segal conjecture regarding the Burnside ring and stable cohomotopy of a finite group $G$ is reduced for the case $G=Z_{2} \times Z_{2}$ to a statement about Ext groups. This statement has since been proved by $H$. Miller, $J$. F. Adams and J. H. C. Gonawardena.
\end{abstract}

The Segal conjecture states that for any finite group $G$, there is an isomorphism $\hat{\alpha}_{G}: A(G) \rightarrow \pi_{s}^{0}(B G)$ from the completed Burnside ring to the zeroth stable cohomotopy group of its classifying space. The conjecture was proved for cyclic groups in [4, 2, and 9]. In this paper we reduce the conjecture for $Z_{2} \times Z_{2}$ to a statement, (1), about Ext groups. This Ext statement was conjectured by Davis in [1] based upon extensive calculations and is proved by Adams, Gunawardena, and Miller in [7].

Let $P=Z_{2}\left[x, x^{-1}\right]$ be made into a module over the mod 2 Steenrod algebra $A$ as in [5], and for $-\infty \leqslant k \leqslant n \leqslant \infty$ let $P_{k}^{n}$ be the subquotient of $P$ which is nonzero in degree $k$ through $n$, inclusive. If $n=\infty$ or $k=-\infty$, they will usually be omitted from the notation. The suspension $\Sigma^{j} M$ of a graded module $M$ is defined by $\left(\Sigma^{j} M\right)_{i+j}=M_{i}$.

STATEMENT 1. There is an epimorphism of $A$-modules

$$
\Sigma P \otimes \Sigma P \stackrel{\phi}{\rightarrow} Z_{2} \oplus \Sigma P_{0}
$$

which induces an isomorphism in $\operatorname{Ext}_{A}\left(, Z_{2}\right)$. In fact

$$
\phi\left(s x^{a} \otimes s x^{b}\right)=\left(\left(\begin{array}{c}
0 \\
a+1
\end{array}\right)\left(\begin{array}{c}
0 \\
b+1
\end{array}\right),\left(\begin{array}{c}
a \\
a+b+1
\end{array}\right) s x^{a+b+1}\right) .
$$

As mentioned above, Statement 1 is proved in [7]. The main result of this paper is

THEOREM 2. Statement 1 implies that $\hat{\alpha}_{Z_{2} \times z_{2}}$ is an isomorphism.

The proof of Theorem 2 mimicks [4]. The main part is to use Statement 1 to calculate $\pi_{s}^{0}\left(R P^{\infty} \times R P^{\infty}\right)$ via the Adams spectral sequence. We begin by deducing from (1) the Ext groups relevant to $\left[R P_{1}^{\infty} \wedge R P_{1}^{\infty}, S^{0}\right]$, the group of stable homotopy classes of maps.

Definition 3. If $M$ is a (left) $A$-module, let $D M$ denote the dual module, made into a left $A$-module using the antiautomorphism $\chi$; i.e., $(D M)_{k}=$ $\operatorname{Hom}_{Z_{2}}\left(M_{-k}, Z_{2}\right)$ with $(\theta \cdot \phi)(m)=\phi(\chi(\theta) m)$.

Received by the editors January 30, 1981.

1980 Mathematics Subject Classification. Primary 55Q10; Secondary 55T15.

Key words and phrases. Segal conjecture, stable cohomotopy groups, Burnside ring.

${ }^{1}$ The author was supported by NSF research grant MCS 7900293. 
PROPOSITION 4. (i) $D\left(P_{k}^{n}\right) \approx \Sigma P_{-n-1}^{-k-1}$ if $-\infty<k<n<\infty$.

(ii) If $M$ has finite type, $\operatorname{Ext}_{A}^{s, t}(L, M) \approx \operatorname{Ext}_{A}^{s, t}(D M, D L)$.

Proof. (i) is well known. If $N$ is a left $A$-module, let $N_{R}$ denote the associated right $A$-module defined using $\chi$. Let $M^{*}$ denote the dual as in [5, 4.3]. Then $\left(N_{R}\right)^{*}=D N$. (ii) follows from $[5,4.3]$ and the symmetry of Tor as follows.

$$
\begin{aligned}
\operatorname{Ext}(L, M) & \approx \operatorname{Ext}\left(L, M^{* *}\right) \approx\left(\operatorname{Tor}\left(M^{*}, L\right)\right)^{*} \\
& \approx\left(\operatorname{Tor}\left(L_{R}, D M\right)\right)^{*} \approx \operatorname{Ext}(D M, D L) .
\end{aligned}
$$

CoRollary 5. $\operatorname{Ext}_{A}^{s, t}\left(Z_{2}, P_{1} \otimes P_{1}\right) \approx \operatorname{Ext}_{A}^{s, t}\left(\Sigma^{2} P^{-2} \otimes P^{-2}, Z_{2}\right)$.

We will also need

Proposition 6. If $M$ is a bounded-above A-module of finite type, then the epimorphism $\Sigma P \rightarrow Z_{2}$ induces isomorphisms

$$
\begin{gathered}
\operatorname{Tor}_{s, t}^{A}\left(Z_{2}, \Sigma P \otimes M\right) \rightarrow \operatorname{Tor}_{s, t}^{A}\left(Z_{2}, M\right), \\
\operatorname{Ext}_{A}^{s, t}\left(M, Z_{2}\right) \rightarrow \operatorname{Ext}_{A}^{s, t}\left(\Sigma P \otimes M, Z_{2}\right) .
\end{gathered}
$$

It was observed in [6] that [5, 1.1] implies Proposition 6, proving it first for finite modules by the 5-lemma, and then observing that $M$ in Proposition 6 is a direct limit of finite modules.

Proposition 7. Statement 1 implies (i) $\operatorname{Ext}_{t}^{s, t}\left(Z_{2}, P_{1} \otimes P_{1}\right)=0$ if $t-s<0$, or if $t=s+1>4$, or if $s=t=0$; (ii) $\operatorname{Ext}_{A}^{1,1}\left(Z_{2}, P_{1} \otimes P_{1}\right)=Z_{2}$; (iii) for $s>2$, $\operatorname{Ext}_{A}^{s, s}\left(Z_{2}, P_{1} \otimes P_{1}\right) \approx Z_{2} \oplus Z_{2}$ with basis $h_{0}^{s-1} a$ and $h_{0}^{s-2} b$.

Proof. We use Corollary 5 to transform into $\operatorname{Ext}\left(, Z_{2}\right)$ and we denote $\operatorname{Ext}_{A}\left(M, Z_{2}\right)$ by $\operatorname{Ext}(M)$. We use the exact sequence

$$
\rightarrow \operatorname{Ext}^{s-1, t}\left(P_{-1}\right) \rightarrow \operatorname{Ext}^{s, t}\left(P^{-2}\right) \rightarrow \operatorname{Ext}^{s, t}(P) \rightarrow \operatorname{Ext}^{s, t}\left(P_{-1}\right) \rightarrow
$$

and Proposition 6 to find

$$
\operatorname{Ext}^{s, t}\left(P^{-2} \otimes P\right)= \begin{cases}Z_{2}, & t-s=-2, s \geqslant 1, \\ Z_{2}, & t-s=-1, s=1,2, \\ 0, & t-s<-2, \text { or } s=0, \text { or } t-s=-1, s>3,\end{cases}
$$

with the $Z_{2}$ 's in $t-s=-2$ related by $h_{0}$ and those in $t-s=-1$ not related by $h_{0}$. Next we use the exact Ext sequence of $P \otimes P_{-1} \rightarrow P \otimes P \rightarrow P \otimes P^{-2}$ together with Statement 1 and the above calculation of $\operatorname{Ext}\left(P^{-2} \otimes P\right)$ to find that in $t-s \leqslant 0$,

$$
\operatorname{Ext}^{s, t}\left(P \otimes P_{-1}\right) \approx \operatorname{Ext}^{s, t}\left(\Sigma^{-1}\left(P_{-1} \oplus P_{0}\right)\right)
$$

To show $\operatorname{Ext}^{s, t}\left(P \otimes P^{-2}\right) \rightarrow \operatorname{Ext}^{s, t}(P \otimes P)$ is nontrivial in this exact sequence when $s=1$ and $t=-1$ and 0 , we use the fact that $\left\{x^{j} \otimes x^{-1}: j \in Z\right\}$ generate $P \otimes P_{-1}$ over $A$ so that $\operatorname{dim}\left(\operatorname{Ext}^{0, t}\left(P \otimes P_{-1}\right)\right) \leqslant 1$.

An easy minimal resolution calculation shows that in $t-s<1, \operatorname{Ext}^{s, t}\left(P_{-1} \otimes P_{-1}\right)$ is zero except for $Z_{2}$ in $(s, t)=(0,-2),(0,-1),(0,0)$, and $(1,2)$. Since $\left\{x^{-1} \otimes x^{j}: j \leqslant-2\right\}$ generate the $A$-module $P_{-1} \otimes P^{-2}, \operatorname{Ext}^{0, t}\left(P_{-1} \otimes P^{-2}\right)=0$ if 
$t \geqslant-2$. These observations and the previous paragraph enable us to calculate the exact Ext sequence of

$$
P_{-1} \otimes P_{-1} \rightarrow P_{-1} \otimes P \rightarrow P_{-1} \otimes P^{-2}
$$

to obtain

$$
\operatorname{Ext}^{s, t}\left(P_{-1} \otimes P^{-2}\right)= \begin{cases}Z_{2}, & t-s=-1, s>1, \\ 0, & t-s<-1, \\ 0, & t-s=0, s>2 .\end{cases}
$$

Finally the Ext sequence of $P_{-1} \otimes P^{-2} \rightarrow P \otimes P^{-2} \rightarrow P^{-2} \otimes P^{-2}$ is easily calculated in this range, yielding the proposition.

Let $\lambda$ denote the usual generator of $\left[R P_{1}, S^{0}\right]$. [4].

COROLlary 8. Statement 1 implies $\left[R P_{1}^{\infty} \wedge R P_{1}^{\infty}, S^{0}\right] \approx Z_{2}^{\wedge} \oplus Z_{2}^{\hat{n}}$ with one summand generated by $\lambda \wedge \lambda$ and the other by a filtration 1 map.

Proof. We filter $R P_{1}^{\infty} \wedge R P_{1}^{\infty}$ by subspaces $X_{l}=R P_{1}^{2 l} \wedge R P_{1}^{2 l}$ so that $\left[X_{l}, S^{0}\right]$ is finite and, hence, lim ${ }^{1}$-terms vanish, and $\left[R P_{1}^{\infty} \wedge R P_{1}^{\infty}, S^{0}\right]$ is a $Z_{2}^{\wedge}$-module as in

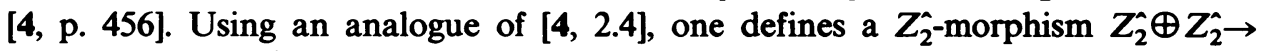
$\left[R P_{1}^{\infty} \wedge R P_{1}^{\infty}, S^{0}\right]$, which is a homeomorphism by the argument of $[4$, p. 456].

Proposition 9. $A\left(Z_{2} \times Z_{2}\right) \approx Z \oplus Z_{2}^{\hat{\imath}} \oplus Z_{2}^{\hat{\imath}} \oplus Z_{2}^{\hat{\oplus}} \oplus Z_{2}^{\hat{2}}$.

Proof. As in [3, pp. 40-47] we find $A\left(Z_{2} \times Z_{2}\right)$ additively isomorphic to a free abelian group on five generators $1, a_{1}, a_{2}, a_{3}, b$, with multiplication $a_{i}^{2}=2 a_{i}$, $a_{i} a_{j}=b$ if $i \neq j$, and augmentation $\varepsilon(1)=1, \varepsilon\left(a_{i}\right)=2, \varepsilon(b)=4$. If $I=\operatorname{ker} \varepsilon$, then $I=\left\langle\left\{2-a_{i}\right\}\right\rangle$, and for $n \geqslant 2, I^{n}=\left\langle\left\{2^{n-1}\left(2-a_{i}\right)\right\}, 2^{n-2}(4-b)\right\rangle$. There is an isomorphism

$$
A\left(Z_{2} \times Z_{2}\right) / I^{n} \stackrel{\phi}{\rightarrow} Z \oplus Z_{2^{n-1}} \oplus Z_{2^{n-1}} \oplus Z_{2^{n-1}} \oplus Z_{2^{n-2}}
$$

defined by

$$
\begin{aligned}
\phi\left(c_{0}+c_{1} a_{1}\right. & \left.+c_{2} a_{2}+c_{3} a_{3}+c_{4} b\right) \\
& =\left(c_{0}+2 c_{1}+2 c_{2}+2 c_{3}+4 c_{4}, c_{1}+c_{2}, c_{2}+c_{3}, c_{3}+2 c_{4}, c_{4}\right) .
\end{aligned}
$$

Proof of Theorem 2. Let $\tilde{A}(G)^{\wedge}$ denote $\operatorname{ker}\left(A(G)^{\wedge} \rightarrow A(1)\right)$. Lin's theorem [4, 1.1] says that $\tilde{A}\left(Z_{2}\right)^{\wedge} \rightarrow\left[R P_{1}^{\infty}, S^{0}\right]$ is an isomorphism of groups isomorphic to $Z_{2}$. There is a commutative diagram with rows split exact, where $\alpha^{\prime}$ is defined by restricting $\hat{\alpha}$ to $\operatorname{ker}(p)$.

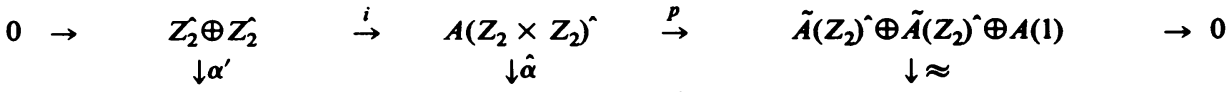

$$
\begin{aligned}
& 0 \rightarrow\left[R P_{1}^{\infty} \wedge R P_{1}^{\infty}, S^{0}\right] \stackrel{i^{\prime}}{\rightarrow}\left[R P_{1}^{\infty} \times R P_{1}^{\infty}, S^{0}\right] \stackrel{P^{\prime}}{\rightarrow}\left[R P_{1}^{\infty}, S^{0}\right] \oplus\left[R P_{1}^{\infty}, S^{0}\right] \oplus\left[S^{0}, S^{0}\right] \rightarrow 0
\end{aligned}
$$

$\hat{\alpha}_{G}$ may be defined by representing a $G$-set $S$ as a homomorphism $G \stackrel{\phi_{s}}{\rightarrow} \delta_{n}$ for some integer $n$ and defining $\hat{\alpha}_{G}(S)$ to be the class of the composite

$$
B G \stackrel{B \phi_{s}}{\rightarrow} B \delta_{n} \rightarrow \underset{j}{\amalg} B S_{j} \stackrel{q}{\rightarrow} \Omega_{0}^{\infty} S^{\infty}
$$


where $q$ is as in [8]. The generators $\tilde{m}$ and $\tilde{w}$ of $Z_{2}^{\hat{2}} \oplus Z_{2}^{\hat{2}}$ satisfy $i(\tilde{m})=[m]-\left[m i_{1}\right]$ $-\left[m i_{2}\right]+2$ and $i(\tilde{w})=[w]-\left[w i_{1}\right]-\left[w i_{2}\right]+4$, where $m: Z_{2} \times Z_{2} \rightarrow \mathcal{S}_{2}$ sends both $T_{1}$ and $T_{2}$ nontrivially, $w: Z_{2} \times Z_{2} \rightarrow \delta_{4}$ satisfies $w\left(T_{1}\right)=\left(\begin{array}{ll}1 & 2\end{array}\right)\left(\begin{array}{ll}3 & 4\end{array}\right)$ and $w\left(T_{2}\right)=\left(\begin{array}{ll}1 & 3\end{array}\right)(24)$, and for $j=1$ and $2, i_{j}: Z_{2} \times Z_{2} \rightarrow Z_{2} \times Z_{2}$ is defined by $i_{j}\left(T_{j}\right)=T_{j}, i_{j}\left(T_{3-j}\right)=0$.

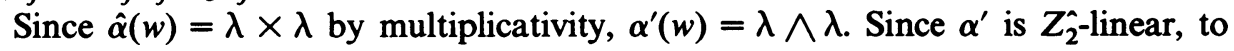
show it is an isomorphism it will suffice to show $\alpha^{\prime}(\tilde{m})$ has filtration 1 . It is well known that if $f$ is the composite

$$
\Sigma^{N} R P^{N-1} \stackrel{\Sigma^{N} \lambda^{\prime}}{\rightarrow} \Sigma^{N} \Omega^{N} S^{N} \stackrel{e}{\rightarrow} S^{N}
$$

then all functional operations $\mathrm{Sq}_{f}^{j} g_{N}$ are nonzero. Since

$$
(B m)^{*}: H^{*} R P^{\infty} \rightarrow H^{*}\left(R P^{\infty} \times R P^{\infty}\right)
$$

sends $x^{i}$ to $\left(x_{1}+x_{2}\right)^{i}, \mathrm{Sq}_{f \circ B m}^{j}\left(g_{N}\right)$ is nonzero in $H^{*}\left(R P^{\infty} \wedge R P^{\infty}\right)$ unless $j-1$ is a 2-power.

\section{REFERENCES}

1. D. M. Davis, On the Segal conjecture for $Z_{2} \times Z_{2}$, Lecture at Oberwolfach Homotopy Theory Conf., Sept., 1980.

2. J. H. C. Gunawardena, Segal's conjecture for cyclic groups of odd prime order, J. T. Knight Prize Essay, Cambridge, 1980.

3. E. Laitinen, On the Burnside ring and stable cohomotopy of a finite group, Math. Scand. 44 (1979), 37-72.

4. W. H. Lin, On conjectures of Mahowald, Segal, and Sullivan, Math. Proc. Cambridge Philos. Soc. 87 (1980), 449-459.

5. W. H. Lin, D. M. Davis, M. Mahowald and J. F. Adams, Calculation of Lin's Ext groups, Math. Proc. Cambridge Philos. Soc. 87 (1980), 459-469.

6. H. Miller, An algebraic analogue of a conjecture of G.W. Whitehead (to appear).

7. J. F. Adams, J. H. C. Gunawardena and H. Miller, The Segal conjecture for $\left(Z_{2}\right)^{n}$ (to appear).

8. S. B. Priddy, On $\Omega^{\infty} S^{\infty}$ and the infinite symmetric group, Proc. Sympos. Pure Math., vol. 22, Amer. Math. Soc., Providence, R.I., 1971, pp. 217-220.

9. D. C. Ravenel, The Segal conjecture for cyclic groups, Bull. London Math. Soc. 13 (1981), 42-44.

Department of Mathematics, Lehigh Universtty, Bethlehem, Pennsylvania 18015 